
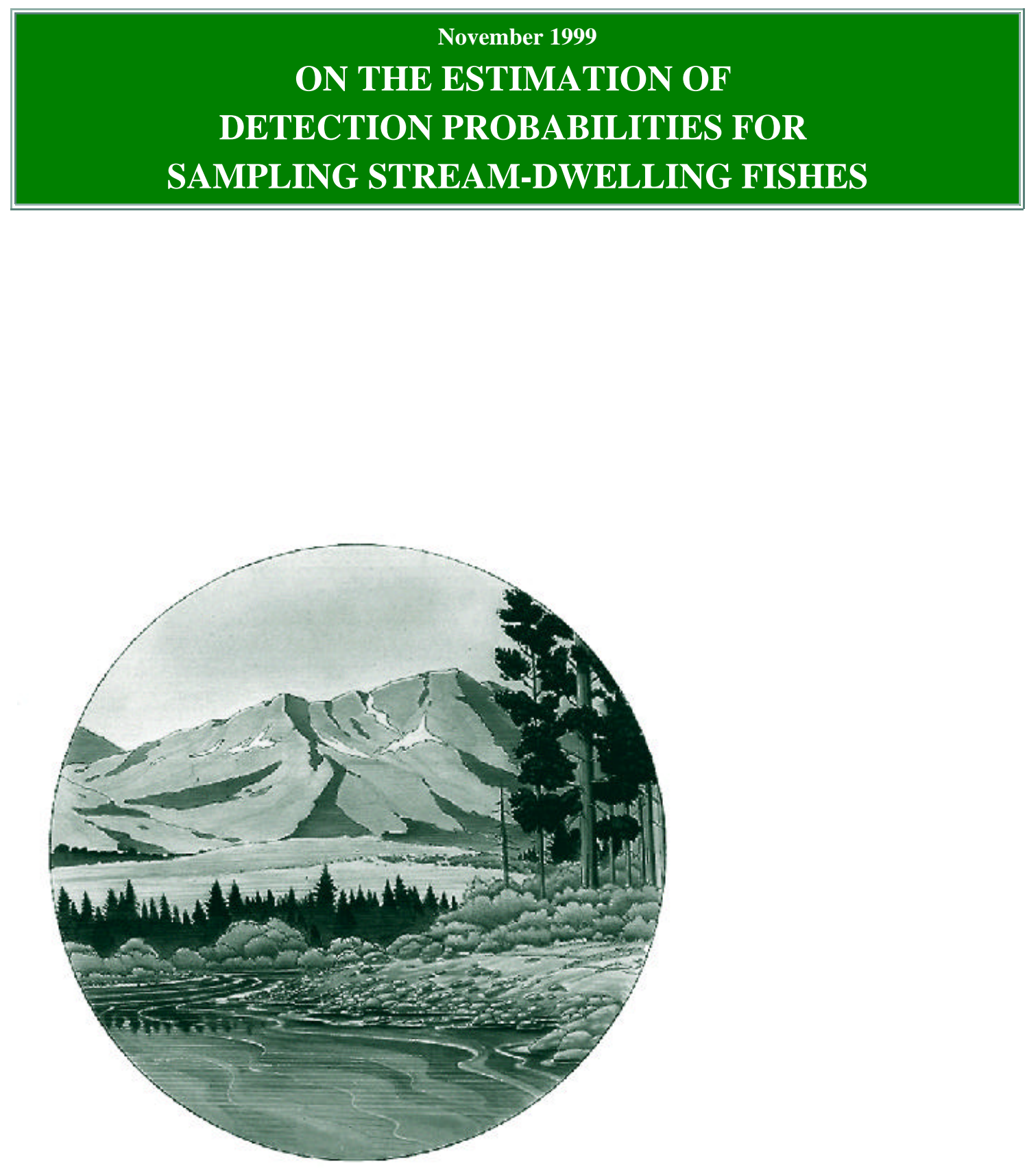

DOE/BP-25866-7 
This report was funded by the Bonneville Power Administration (BPA), U.S. Department of Energy, as part of BPA's program to protect, mitigate, and enhance fish and wildlife affected by the development and operation of hydroelectric facilities on the Columbia River and its tributaries. The views of this report are the author's and do not necessarily represent the views of BPA.

This document should be cited as follows:

Peterson, James T. - U.S. Department of Agriculture Forest Service, On the estimation of detection probabilities for sampling stream-dwelling fishes, Report to Bonneville Power Administration, Contract No. 1992AI25866, Project No. 199203200, 40 electronic pages (BPA Report DOE/BP-25866-7)

This report and other BPA Fish and Wildlife Publications are available on the Internet at:

\section{http://www.efw.bpa.gov/cgi-bin/efw/FW/publications.cgi}

For other information on electronic documents or other printed media, contact or write to:

Bonneville Power Administration

Environment, Fish and Wildlife Division

P.O. Box 3621

905 N.E. 11th Avenue

Portland, OR 97208-3621

Please include title, author, and DOE/BP number in the request. 


\title{
On the estimation of detection probabilities for sampling stream-dwelling fishes
}

\author{
Prepared by: \\ James T. Peterson \\ U.S. Department of Agriculture Forest Service, \\ Rocky Mountain Research Station, Boise, Idaho
}

Prepared for:

U. S. Department of Energy

Bonneville Power Administration

Environment, Fish and Wildlife

P.O. Box 3621

Portland, OR 97208-3621

Project Number 92-032-00

Contract Number 92AI25866

November 1999 


\section{TABLE OF CONTENTS}

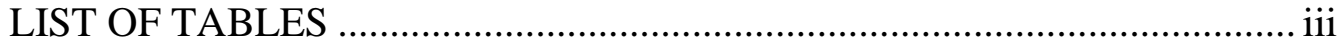

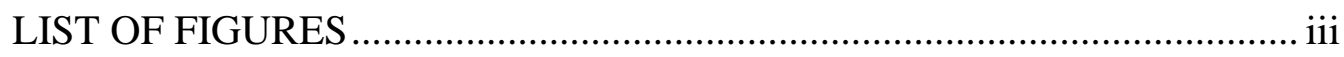

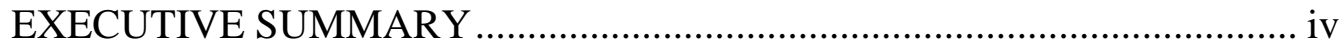

INTRODUCTION

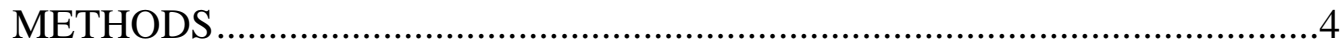

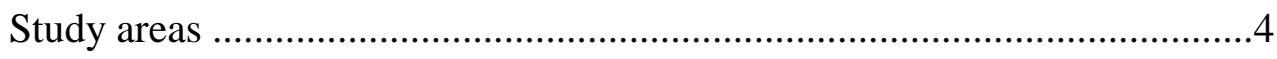

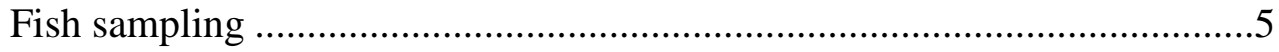

Definitions and statistical analysis ............................................................

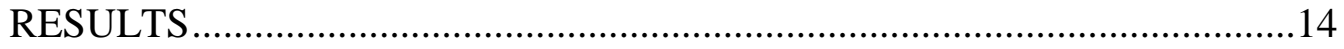

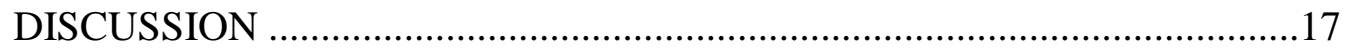

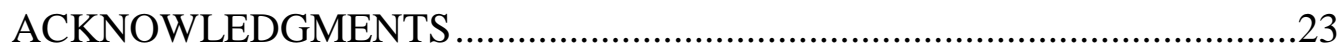

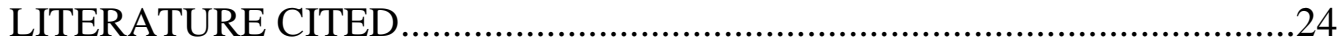




\section{List of Tables}

Table

1. Means, standard errors and range of mean density and the negative binomial dispersion parameter, by species, and coefficients, standard errors, and $95 \%$ confidence limit from linear regression of species-specific mean abundance and negative binomial dispersion parameter

2. Average habitat characteristics, fish abundance, and relative sampling efficiency for monitoring sites used for the comparison of 4 approaches to estimating detection probabilities, for bull and westslope cutthroat trout.

3. Mean density, variances, gamma shape and scale parameters, negative binomial dispersion parameter, probability of detection estimates, and sample size requirements for $95 \%$ power, by species, for salmonids collected during FS survey of small streams in the Interior Columbia River

Basin.

\section{List of Figures}

Figure

1. Locations of surveyed and monitored stream channels (gray lines) in the interior Columbia River Basin (bold outline). States are labeled and boundaries (broken line) are shown for reference .32

2. Abundance estimates from the quasi-likelihood Poisson and negative binomial regressions for (a) bull trout, (b) brook trout, and (c) westslope cutthroat trout calculated during the leave-one-out cross-validation procedure. Broken line represents perfect fit.

3. The mean differences between baseline detection probabilities and four detection probability estimators for bull trout, brook trout, and westslope cutthroat trout 


\section{Executive Summary}

To examine the adequacy of fish probability of detection estimates, I examined distributional properties of survey and monitoring data for bull trout (Salvelinus confluentus), brook trout (Salvelinus fontinalis), westslope cutthroat trout (Oncorhynchus clarki lewisi), chinook salmon parr (Oncorhynchus tshawytscha), and steelhead /redband trout (Oncorhynchus mykiss spp.), from 178 streams in the Interior Columbia River Basin. Negative binomial dispersion parameters varied considerably among species and streams, but were significantly $(\underline{\mathrm{P}}<0.05)$ positively related to fish density. Across streams, the variances in fish abundances differed greatly among species and indicated that the data for all species were overdispersed with respect to the Poisson (i.e., the variances exceeded the means). This significantly affected Poisson probability of detection estimates, which were the highest across species and were, on average, 3.82, 2.66, and 3.47 times greater than baseline values. Required sample sizes for species detection at the 95\% confidence level were also lowest for the Poisson, which underestimated sample size requirements an average of $72 \%$ across species. Negative binomial and Poissongamma probability of detection and sample size estimates were more accurate than the Poisson and generally less than $10 \%$ from baseline values. My results indicate the Poisson and binomial assumptions often are violated, which results in probability of detection estimates that are biased high and sample size estimates that are biased low. To increase the accuracy of these estimates, I recommend that future studies use predictive distributions than can incorporate multiple sources of uncertainty or excess variance and that all distributional assumptions be explicitly tested. 


\section{Introduction}

The development of efficient and effective stream-fish management strategies requires the analysis of high quality data. However, many fish species are difficult to sample due to physical (e.g., morphology) and behavioral (e.g., habitat use) characteristics or are difficult to locate because they are relatively rare (Rieman and McIntyre 1995; Bonar et al 1997; Watson and Hillman 1997). In these instances, data collection and analyses often are limited to species presence and absence (Strayer 1999). The ability to detect a species is, in part, positively related to the amount of sampling effort (e.g., sample size, Elliot 1969; Pielou 1969; Poole 1974). Collecting too few samples can affect the quality of presence and absence data by lowering the ability to detect a species when present (i.e., false absence), whereas collecting too many samples is not cost-effective and wastes resources. To maintain consistent levels of effort, sample size requirements should be determined prior to developing sampling protocols.

For presence and absence studies, sample size requirements are estimated using species-specific estimates of the probability of detecting the species in a single sample (Elliot 1969; Pielou 1969; Poole 1974). Probability of detection estimates, in turn, require estimates of fish abundance and distribution that are best approximated by a discrete statistical distribution (i.e., a sampling unit cannot contain a fraction of a fish). Previous studies have modeled organismal abundance and distribution using the Poisson and negative binomial distributions (Bliss and Fisher 1953; Green and Young 1993; Watson and Hillman 1997). Although both distributions are discrete and can range from zero to infinity, they fundamentally differ in how they model variance and approximate species distributions. The Poisson distribution has one parameter $(\underline{m})$ that is both the mean and 
the variance and hence, it assumes that the mean and variance are equal. In contrast, the negative binomial has two parameters, the mean $(\underline{\mathrm{m}})$ and dispersion $(\underline{\mathrm{k}})$, which is a measure of variability. Somewhat counter-intuitively, low values of $\underline{\mathrm{k}}$ indicate high dispersion (variance) and high values indicate low dispersion. As $\underline{\mathrm{k}}$ gets very large, the negative binomial is equivalent to the Poisson (Pielou 1969; Poole 1974). Thus, variance can be less than or greater than the mean for the negative binomial distribution.

For a fixed sampling unit size, the negative binomial dispersion parameter $(k)$ has been biologically interpreted as the degree of clumping in a population's distribution (Elliot 1969; Pielou 1969; Poole 1974). Small values of $k$ indicate a very clumped distribution and large values indicate a more random distribution. For a fixed mean, $k$ also can be thought of as an index of the number of groups (clumps) containing all of the individuals in a population (Southwood 1966), which affects the probability of detecting the population. For example, consider a population consisting of 10 individuals and 10 areas (sample units) where the individuals can occur. If each area contains 1 individual (all areas occupied), the mean density would be 1 and the single sample probability of detection would be $100 \%$. If all 10 individuals were in a single area ( 1 clump), the mean density would still be 1 but the single sample probability of detection would be $1 / 10$ or $10 \%$. In contrast to the negative binomial, the Poisson assumes that individuals in a population are randomly distributed and also assumes spatial independence (Pielou 1969). That is, the distributions of individuals in a population are independent of one another and other factors, such as physical habitat characteristics. Fish distribution, however, is nonrandom and can be influenced by landscape (Matthews 1988; Thurow et al. 1997; Dunham and Rieman. 1999) and physical habitat characteristics (Gorman and 
Karr 1978; Angermeier and Karr 1984; Bisson et al. 1988; Baltz et al 1991) and intraand inter-specific interactions (Edmundsen et al. 1968; Fraser et al. 1987; Baltz et al. 1982; Bisson et al. 1988). Thus, it is likely that the Poisson assumptions cannot be met, limiting its usefulness for estimating fish detection probabilities and sample size requirements.

Previous studies have justified the use of the Poisson to estimate detection probabilities for rare species by assuming that there is a negative relationship between a species' density and dispersion, $\underline{\mathrm{k}}$ (Green and Young 1993; Bonar et al 1997). That is, a species' distribution becomes more random (less clumped) as it becomes rare. Although there is some evidence for this relationship for relatively sessile organisms (Green and Young 1993; Elliot 1969; see however Hariston 1959), the validity of this assumption has yet to be evaluated for fishes.

Both the negative binomial and Poisson estimators require that all individuals in a sampling unit can be counted (Elliot 1969; Pielou 1969; Poole 1974). In practice, this is impossible for most fish collection efforts because fish sampling efficiency is rarely, if ever, 100\% (Reynolds 1996; Dolloff et el. 1996 and references therein). Previous efforts have attempted to account for the influence of sampling efficiency by assuming that fish capture is a binomial process (Rieman and McIntyre 1995; Bonar et al.1997). That is, a fish is either captured or missed (e.g., a fraction of a fish cannot be caught). Detection probabilities then were adjusted assuming a binomial distribution and using a point estimate of sampling efficiency $(q)$. The binomial adjustment assumes that all individuals within a sampling unit have the same probability of capture and all respond independently. However, sampling efficiency is influenced by body size (Buttiker 1992; 
Anderson 1995; Dolloff et el. 1996) and thus, it likely varies among individuals within a sampling unit. The prevalence of overdispersion (variance in excess of the binomial distribution) in many fish sampling efficiency and mark and recapture models also suggests that fish do not respond independently (Bayley 1993). Therefore, the binomial assumptions are likely violated.

The use of the Poisson and binomial (for adjustment) distributions to estimate detection probabilities and sample size requirements are gaining widespread acceptance (Hillman and Platts 1993; Rieman and McIntyre 1995; Bonar et al. 1997; Watson and Hillman 1997; Dunham and Rieman 1999). Unfortunately, there have been no studies examining validity of their assumptions and the effects of potential violations. Thus, I examined the adequacy of several methods for estimating detection probabilities with the following objectives: (1) determine the relationship between density and negative binomial dispersion for several fish species, (2) examine the influence of dispersion on probability of detection and sample size estimates, (3) determine the relative accuracy of probability of detection estimates four composite estimators, and (4) provide recommendations for the development of future sampling strategies.

\section{Methods}

I examined the adequacy of several methods for estimating fish detection probabilities using 2 sets of data: one was collected for a large-scale fish and fish habitat survey and the other for monitoring trends in fish population sizes. In all applications, trained personnel collected fish with standardized protocols from various streams types. Assuming that most fish sampling and monitoring are conducted under similar circumstances, I considered these data typical of data that are collected when the 
probability of detection is a concern (e.g., monitoring rare and endangered species). I caution, however, that this is an analysis of 'found data' (sensu Overton et al. 1993). That is, the data were collected for a variety of purposes and site selection was nonrandom. Therefore, the reported detection probability estimates should not be extrapolated or applied to other systems. Rather, these estimates should only be used to compare the relative usefulness of the various methods.

\section{$\underline{\text { Study Areas and Fish Sampling }}$}

Forest Service Survey.- Fishes were surveyed by USDA Forest Service (FS) biologists during the summer months in 156 stream reaches within the Columbia River Basin from 1991- 96 (Peterson and Wollrab 1999). Most of the reaches were located in Central Idaho and Western Montana (Figure 1), with the majority of these in the Salmon River Basin, Idaho. Surveys were generally conducted to provide baseline data before the initiation of management activities, such as timber harvest or cattle grazing (Peterson and Wollrab 1999), or to collect reference data on natural or desired conditions (Overton et al. 1995). Consequently, they represent a wide variety of stream types and habitats encountered in the Basin.

All fishes were sampled via daytime snorkeling within individual sampling units that were deep enough to submerge a diver's mask. Sampling unit selection procedures were nonrandom, varied among reaches, and depended upon the decisions of the field biologists. In general, the biologists attempted to sample the habitats most likely to contain salmonids (e.g., pools). All sampling began at the downstream end of a sampling unit and 1-2 divers swam upstream, counted each fish observed, and 
identified all salmonids to species. Note that steelhead parr could not be distinguished from sympatric redband trout and the data were combined into a single group.

Fish monitoring data.- Fishes were monitored by Idaho Department of Fish and Game, USDA Forest Service, Nez Perce Tribe and Shoshone-Bannock Tribes during the summer months at 838 established sites between 1984-97 (Rieman et al 1999). These sites were distributed across the Salmon and Clearwater River Basins in Central Idaho (Figure 1) and were chosen to provide an annual index of chinook salmon and steelhead production. Relative abundance data for bull trout, brook trout, and westslope cutthroat trout also were collected during sampling.

All sampling was conducted via daytime snorkeling at the established sites (average 100m long) and samples were generally collected during low-flow conditions. Sampling began at the downstream end of each site and 1-2 divers swam upstream, counted each fish, and identified salmonids to species. Definitions and Statistical Analysis

Density and dispersion.- A fundamental assumption of using the Poisson estimator for rare species is that there is a negative relationship between a species' density and dispersion (Green and Young 1993). Unfortunately, this relationship cannot be examined with fish collection data because the statistical distribution (i.e., dispersion) of fish collection data is a function of a species' spatial distribution and the variability in sampling efficiency. An examination of raw (unadjusted) catch data, however, could provide some insight into combined effect of fish spatial distribution and sampling variability on data dispersion and its influence on detection estimates. To examine the relationship between data dispersion and density estimates, I selected 58 streams that 
were known to contain populations of 5 salmonid species (Table 1) from the FS Survey database. The analysis was restricted to smaller streams (average wetted width 3-6 m) to maintain a relatively constant sample unit area and to minimize the potential differences in sampling efficiency. (Note that I included all of the streams in the FS database that were in the known range of all 5 species and that met the size criteria.) Stream reach lengths averaged $7.2 \mathrm{~km}$; sample unit lengths, $10.1 \mathrm{~m}$; and the number sample units per stream reach varied between 10-54. Therefore, the proportion of each stream reach sampled was very small and I assumed an infinite sample size in which no finite population correction factor was needed.

Means and variances were estimated for each stream by species combination and the negative binomial distribution parameter, $\underline{\mathrm{k}}$, was fit via maximum likelihood (Poole 1974). In several instances, a dispersion parameter could not be estimated because a species was not collected in a surveyed stream (Table 1). The dispersion parameter, $\underline{\mathrm{k}}$, also showed a large range of values within and among species (Table 1) and was natural $\log$ transformed prior to regression analysis (below).

The relationship between mean density and the dispersion parameter was examined via linear regression. Differences among species were examined by recoding species identities as dummy variables (i.e., 0 or 1 ) with steelhead/ redband trout as the baseline because it had the fewest missing observations (i.e., a dispersion parameter could be estimated for the greatest number of sites). Goodness-of-fit was assessed for the regression model by examining residual and normal probability plots (Nelder et al. 1993). Ninety-five percent confidence intervals were constructed for each parameter estimate to provide a means to assess precision and statistical significance. Note that the mean and 
dispersion parameter estimates were estimated with error, but the effect of this error on the regression should only result in a weaker relationship between the mean density and dispersion (Green and Young 1993).

Prior and predictive distributions.- Assuming a Poisson distribution, the probability ( $\underline{\underline{e}}_{\mathrm{e}}$ ) of encountering (not necessarily capturing) at least 1 individual in a single sample (site) is one minus the probability of encountering no individuals $(\underline{\mathrm{i}}=0)$ and is estimated as:

$$
p_{e}=1-\left(m^{i} \frac{e^{-m}}{\Gamma(i+1)}\right)
$$

where $\underline{m}$ is mean or expected number of individuals per sampling unit. This estimate, however, only takes into account process uncertainty (i.e., the uncertainty associated with spatial and temporal fish distribution, given $\underline{\mathrm{m}}$ ) and assumes a fixed or known $\underline{\mathrm{m}}$. Another potentially significant source of uncertainty (variance) is that which is associated with the value of $\underline{m}$, referred to as the parameter uncertainty. Parameter uncertainty represents the variability of $\underline{\mathrm{m}}$ (e.g., among locations or through time). Fishery biologists are used to expressing this source of variability with some measure of precision, such as confidence intervals or standard errors. To account for parameter uncertainty, $\underline{\mathrm{m}}$ can be treated as a random variable with its own distribution and mean. For the Poisson, the natural conjugate distribution (also referred to as the prior) is the generalized gamma (Berger 1985):

$$
f(m)=\frac{1}{\Gamma(a)}\left(\frac{1}{b}\right)^{a}\left[m^{a-1} e^{-\frac{m}{b}}\right]
$$

where $\underline{a}$ and $\underline{b}$ are the shape and scale parameters, respectively. Assuming that all of the parameters $(\underline{a}, \underline{b}, \underline{m})$ are greater than zero, the variation in $m$ then can be incorporated by 
combining the gamma and the Poisson and integrating over $\underline{m}$ as:

$$
p_{e}=1-\int_{0}^{\infty} \operatorname{Pr}(I=i \mid m) f(m) d m,
$$

to obtain the predictive distribution of the Poisson, the negative binomial. The probability of encountering at least 1 individual then is estimated as:

$$
p_{e}=1-\left[\frac{b^{i}}{(1+b)^{a+i}} \frac{\Gamma(a+i)}{\Gamma(i+1) \Gamma(a)}\right] \text {, }
$$

where $a$ is the dispersion parameter $(\underline{\mathrm{k}}), \underline{\mathrm{b}}$ is equivalent to the ratio of the negative binomial parameters ( $\underline{\mathrm{m}} / \underline{\mathrm{k}})$, and $\underline{\mathrm{i}}=0$ as defined above (Pielou 1966).

One of the difficulties with using the negative binomial to estimate detection probabilities and sample size requirements is the relatively large amount of data needed to estimate both the mean and dispersion parameter (Green and Young 1993). The gamma could provide the means to incorporate existing information on the inherent variability of $\underline{\mathrm{m}}$. To examine usefulness of a gamma prior, I compared the probability of detection estimates for Poisson, Poisson-gamma (i.e., negative binomial with gamma parameters), and negative binomial estimators using the FS survey data described above. Mean densities and maximum likelihood estimates of the gamma and negative binomial parameters were estimated for each species, across streams, and goodness-of-fit was assessed via chi-square tests (Sokal and Rohlf 1995). Probabilities of detection were estimated using the maximum likelihood estimates and equations 1 and 4, with $\underline{b}$ replaced by $\underline{\mathrm{m}} \underline{\mathrm{k}}$ in equation 4 for the negative binomial estimates. These probabilities were then used to estimate the number of samples required for species detection at the $95 \%$ confidence level according to Green and Young (1993). 
Sampling efficiency and variability.- For clarity, I define sampling efficiency as the proportion of individuals, in a given area, that are captured or observed during sampling. The effect of sampling efficiency on detection can be estimated by assuming capture is a binomial process and that individuals respond independently and are equally catchable. Thus, the probability of capturing $\left(\underline{p}_{\mathrm{c}}\right)$ at least 1 individual, given the sampling efficiency (q) and number of individuals encountered (i), is one minus the probability of capturing no individuals and is estimated as:

$$
\underline{\mathrm{p}}_{\mathrm{c}}=1-(1-\underline{\mathrm{q}})^{\underline{\mathrm{i}}}
$$

Similar to the Poisson estimator, the binomial only takes into account process uncertainty and assumes a fixed or known q. The uncertainty or variability of q (e.g., due to differences in sampling efficiency among individuals) can be taken into account by treating $q$ as a random variable with a beta distribution, which results in predictive distribution of the binomial, the beta-binomial (Berger 1985). The probability of capturing at least 1 individual is then estimated as:

$$
\mathrm{p}_{\mathrm{c}}=1-\left[\frac{\Gamma(i+1) \Gamma(a+x) \Gamma(a+b) \Gamma(i+b-x)}{\Gamma(x+1) \Gamma(i-x+1) \Gamma(a+b+i) \Gamma(a) \Gamma(b)}\right],
$$

where $\underline{\mathrm{a}}$ and $\underline{\mathrm{b}}$ are the beta shape parameters and $x$ is the number of individuals captured ( 0 in this case). The assumption of independence among individuals is also relaxed for the beta-binomial (Prentice 1986).

Relative accuracy evaluation.- Evaluating the 'true' accuracy of detection estimates requires species' presence and absence to be known with certainty. Unfortunately, these estimates are generally unobtainable unless study areas are stocked with known numbers of (presumably marked) individuals, which could be costprohibitive when conducted over the large number of sites presumably needed to obtain 
reliable estimates. An alternative approach (and the one used here) is to use data for sites that were sampled on multiple occasions and assume that occupied sites are always potentially occupied (i.e., during sampling, across occasions). The proportion of sampling occasions that a species was detected could provide a baseline for comparing the relative accuracy of various estimation techniques. Thus, I examined the relative accuracy of 4 detection probability estimators (outlined below) using the fish monitoring data (Rieman et al 1999) for occupied sites that were sampled on 10 or more occasions. This cutoff was selected for practical reasons, such as resolution (e.g., only 3 relative efficiency estimates are possible for 3 sampling occasions, $0.33,0.67$ and 1.00), and to ensure that sites with high probabilities of detection were not over represented. The analysis was also restricted to bull trout, brook trout, and westslope cutthroat trout because the densities of chinook salmon parr and steelhead/redband trout were confounded by supplementation (stocking), which would likely affect the accuracy of fish abundance models.

The probability of detecting a fish species is a function of sampling efficiency (i.e., probability of capture, $\underline{\underline{p}}_{\mathrm{c}}$ ) and the number of vulnerable fish (i.e., probability of encounter, $\underline{p}_{\mathrm{e}}$ ), both of which are influenced by habitat features. Thus, to estimate the probability of detection requires site-specific estimates of sampling efficiency and fish abundance. Sampling efficiencies were estimated for each site and sampling occasion using beta-binomial models of relative sampling efficiency (Reiman et al. 1999; R. Thurow, Rocky Mountain Research Station 316 E. Myrtle Street Boise Idaho, unpublished data) and were used to adjust fish abundance estimates. Site-specific estimates of sampling efficiency, fish abundance, and habitat characteristics were then averaged to approximate "average" sampling conditions and abundance, across sampling 
occasions (Table 2). Abundance estimates were rounded to the nearest integer to facilitate model fitting with a discrete distribution.

The relative accuracy of detection estimates was assessed via leave-one-out crossvalidation for 4 combinations of the probability of encounter $\left(\underline{\underline{p}}_{\mathrm{e}}\right)$ and capture $\left(\underline{\mathrm{p}}_{\mathrm{c}}\right)$ estimators described above: the Poisson-binomial (P-B), Poisson-beta-binomial (P-BB), negative binomial-binomial (NB-B) and negative binomial-beta-binomial (NB-BB). During the cross-validation procedure, one observation (site) was excluded from the data set; Poisson or negative binomial regression models (White and Bennetts 1996) were fitted to the remaining data with fish abundance as the response and site dimensions, depth, gradient, and elevation as the predictors; and the regression model was used to predict fish abundance and the dispersion parameter (for the negative binomial) for the excluded observation. The probability of detection, $\underline{\operatorname{Pr}}(\underline{\text { detect }})$, was then estimated for the left-out observation as:

$$
\underline{\operatorname{Pr}}(\underline{\text { detect }})=\sum_{i=0}^{\infty}[\operatorname{Pr}(X>0 \mid i, q) \operatorname{Pr}(I=i \mid m)]
$$

where, for the Poisson- binomial, $\underline{\operatorname{Pr}}(\underline{\mathrm{I}}=\underline{\mathrm{i}} \mid \underline{\mathrm{m}})$ is the probability of encountering $\underline{\mathrm{i}}$ individuals assuming a Poisson distribution (equation 1), given the estimated mean abundance $(\underline{\mathrm{m}})$ from the Poisson regression, and $\underline{\operatorname{Pr}}(\underline{X}>0 \mid \underline{\mathrm{i}}, \underline{q})$ is the probability of capturing at least 1 individual, given the number of individuals encountered and the estimated sampling efficiency (equation 5). Combinations that included the negative binomial and beta-binomial used equations 4 and 6 in place of equations 1 and 5, respectively. The beta-binomial shape parameters were estimated as $\underline{a}=\underline{q} / \gamma$ and $\underline{b}=(1-\underline{q}) / \gamma$, where $\gamma$ is the dispersion parameter from the beta-binomial regression model 
that estimates the sampling efficiency variability (i.e., larger values indicate greater sampling variability). Note that the term within the brackets above is a Taylor expansion (i.e., it converges to a value as $i \rightarrow \infty$; Hillborn and Mangel 1997). During the crossvalidation, the summation converged rapidly and hence, $i$ did not exceed 500 in practice.

Prior to the cross-validation procedure, Poisson and negative binomial regressions were fitted to the full data set and goodness-of-fit was assessed for each with chi-square tests (Sokal and Rohlf 1995). The similarity between the Poisson and negative binomial site-specific abundances estimates also were examined for each species via Pearson correlations. The relative accuracy of each of 4 composite estimators (P-B, P-BB, NB-B, and NB-BB) was assessed as the difference between site-specific probability of detection estimates and the baseline (i.e., the proportion of sampling occasions detected). Ninetyfive percent confidence intervals of differences were calculated following Zar (1996).

\section{Results}

Density and dispersion.- Negative binomial dispersion parameters could not be calculated for each stream by species combination because in several instances a species was not collected in a surveyed stream (Table 1). Of the streams where individuals were collected, mean density and the negative binomial dispersion parameters varied considerably among species and streams (Table 1). Densities were greatest for chinook salmon parr and steelhead/redband trout and lowest for bull trout. Across species, fish were rare in $12 \%$ of the 58 streams (densities < 0.1 as defined by Green and Young 1993). The negative binomial dispersion parameters were generally low and did not exceed 1 for $53 \%$ of the observations (low values indicate greater clumping or overdispersion). However, 4 of the dispersion estimates, 1 steelhead/rainbow trout and 3 
bull trout, exceeded 100. An examination of the data indicated that they were collected in accordance with the protocols and that the sampling conditions and habitat was similar to the other streams. Therefore, there was no basis for their removal from the data and they were included in the regression.

Regression analysis indicated a statistically significant $(\underline{\mathrm{P}}<0.05)$ positive relationship between raw density and data dispersion across species (i.e., overdispersion increased as density decreased; Table 1). Differences among species varied widely and chinook salmon was the only species to differ significantly $(\underline{\mathrm{P}}<0.05)$ from steelhead/rainbow trout (the baseline). Density by species interactions also varied considerably in magnitude and direction (Table 1), but none were statistically significant $(\underline{\mathrm{P}}>0.05)$.

$\underline{\text { Prior and predictive distributions.- Variances differed greatly among species and }}$ indicated that the catch data for all species were overdispersed with respect to the Poisson (i.e., the variances exceeded the means; Table 3). This significantly affected Poisson probability of detection estimates, which were the highest across species and were, on average, 3.82, 2.66, and 3.47 times greater than the baseline, Poisson-gamma, and negative binomial, respectively (Table 3). Required sample sizes for species detection at the $95 \%$ confidence level also were lowest for the Poisson, which underestimated sample size requirements an average of $72 \%$, across species. Negative binomial dispersion parameters also varied among species, which suggested that chinook salmon parr distribution patterns were the most clumped among species. The negative binomial probability of detection and sample size estimates were the closest to, and differed from the baseline an average of $9.6 \%$ and $9.5 \%$, respectively. Poisson-gamma probability of 
detection and sample size estimates were similar to the negative binomial except for brook trout and chinook salmon, which were the only species to fail goodness-of-fit tests for the gamma $(\underline{\mathrm{P}}<0.05)$. Nonetheless, these estimates were much closer to the baseline than the corresponding Poisson estimates (Table 3).

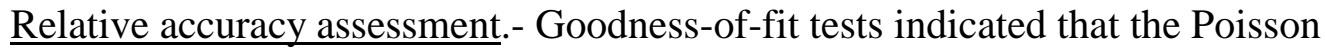
regression model fitted to the full data set were overdispersed for all species (i.e., the variance exceeded the presumed Poisson). Quasi-likelihood Poisson regression is similar to Poisson regression, but it has an additional element to account for error variances in excess of assumed distributions (Wang et al. 1996). Therefore, I fit quasi-likelihood Poisson regression models for the leave-one-out cross-validation procedure.

Pearson correlations of site-specific abundances estimated during the crossvalidation indicated that quasi-likelihood Poisson and negative binomial estimates were very similar for bull trout $(\underline{\mathrm{r}}=0.98)$, brook trout $(0.94)$, and westslope cutthroat trout (0.95). On average, quasi-likelihood Poisson estimates differed from their negative binomial counterparts the by $8 \%$ across species (Figure 2).

Probability of detection estimates indicated significant differences among the 4 composite predictors across species (Figure 3). On average, bull trout, brook trout, and westslope cutthroat trout were detected at multiply-sampled, occupied sites on $33.7 \%$, $40.1 \%$, and $46.6 \%$ of sampling occasions, respectively. The NB-BB detection estimates were the most accurate (relative) for all species and were not significantly different $(\mathrm{P}>0.05)$ from the baseline (Figure 3). On average, bull trout, brook trout, and westslope cutthroat trout NB-BB detection estimates differed from the relative detection probabilities by $0.01,-0.03$ (underestimate), and 0.05 , respectively. In contrast, the P-B 
estimates were the least accurate and significantly $(\underline{\mathrm{P}}<0.05)$ overestimated detection probabilities by $0.28,0.32$, and 0.47 for bull trout, brook trout, and westslope cutthroat trout relative, respectively. In contrast to the other species, the brook trout NB-B estimates also were not significantly different from the baseline (Figure 3). The relative accuracy of the estimators also differed among species. The bull trout P-BB estimates were more accurate than the NB-B, whereas the opposite pattern was observed for the other two species (Figure 3).

\section{Discussion}

The variability of fish density and sampling efficiency had a profound effect on the relative accuracy of the probability of detection estimates. The negative binomial and beta-binomial estimators were consistently more accurate than the Poisson and binomial. These discrepancies were not due to differences in fish density or sampling efficiency estimates. Poisson and negative binomial estimators used the same or similar fish densities (Table 3; Figure 3), yet the Poisson detection estimates were as much as 584\% greater than the negative binomial estimates (Table 3). Similarly, the binomial and betabinomial estimators used the same sampling efficiency estimates (Table 2) and binomial detection estimates were, on average, $26 \%$ greater than their beta-binomial counterparts (Figure 2). The major difference between these estimators is their ability to incorporate additional variability in fish density or sampling efficiency. The negative binomial and beta-binomial estimators explicitly incorporate extra variation (or uncertainty) via the dispersion parameters, whereas the Poisson and binomial cannot (Gelman et al. 1995). Consequently, the Poisson and binomial almost certainly underestimated the total variation associated with fish sampling, which resulted in overly optimistic (i.e., biased 
high) estimates of probability of detection. To increase the accuracy of probability of detection estimates, I recommend that future studies use predictive distributions that can incorporate multiple sources of uncertainty or additional variance.

Variance also had a significant effect on sample size estimation. The Poissonbased sample size estimates for $95 \%$ detection level were, on average, $72 \%$ lower than would have been required for (baseline) detection (Table 3), whereas the negative binomial estimates were 9.6\% lower. Interestingly, Watson and Hillman (1997) assumed that bull trout were distributed as a Poisson with a mean density ( 0.25 per sampling unit) identical to that of the FS survey data (Table 3), and estimated that 12 samples assured detection probabilities of $95 \%$. Although they did not provide variance estimates to determine if their data were overdispersed, the prevalence of overdispersion (relative to the Poisson) in the FS survey and fish monitoring data suggest that it was likely. In fact, biological count data (e.g., fish abundance) are more often distributed as a negative binomial (overdispersed) than a Poisson (White and Bennetts 1996). Failure to incorporate extra variance would result in the systematic underestimation of required sample sizes. For example, the FS survey data suggest that Watson and Hillman might have underestimated the required sample sizes by one third, potentially missing bull trout when they were present. Inadequate sampling effort (number of samples) would likely result in mistaken or false absences, which would waste valuable management resources and potentially place species at risk. To avoid such mistakes, I urge researchers to rigorously evaluate their assumptions and further suggest that journal editors and reviewers insist that results of such evaluations be reported. 
Negative binomial dispersion was positively related to (raw) density for the 5 salmonid species considered (Table 1), which suggested that species' distributions became more clumped as a species became rarer. This was contrary to the reported patterns for some benthic aquatic invertebrates (Elliott 1969; Green and Young 1993) and plants (Greig-Smith 1964), but is consistent with Harrison's (1959) analysis of soil arthropods. These conflicting studies, however, were conducted on relative sessile organisms. Because dispersion patterns are influenced by species behavior (Poole 1974), these differences were likely due, in part, to the greater mobility of fishes. Streamdwelling salmonids can move relatively long distances to exploit newly created habitats or food resources (Gowan et al. 1994 and references therein) and will congregate where resources are abundant. Fish also can vary their distributions patterns (i.e., move) seasonally, within and among reaches, in response to changes in the distribution of prey species (Petty and Grossman 1996). Schooling behavior also can influence dispersion. Small fish schools often combine in order to maintain a critical size (Shaw 1978), which would cause the population to become more clumped as the number of schools decreased. Among fish species, distribution patterns also were influenced, in part, by speciesspecific behaviors. For instance, the negative binomial dispersion parameter was lowest for chinook salmon parr, a species generally found in schools (Scott and Crossman 1973), whereas it was greatest for steelhead/ redband trout, a somewhat territorial species (Edmundson et al. 1968). Fish distribution patterns also are influenced by environmental heterogeneity, which can operate independent of density. For example, the distributions of several warmwater streams fishes are significantly influenced by the juxtaposition of tributaries (Osborne and Wiley 1992). The relatively large differences among species 
dispersion parameters (Tables 1 and 3) also suggest that no single dispersion parameter would likely be adequate for estimating detection probabilities and developing sampling guidelines, across several fish species. Rather, the best approach would be to use existing sampling data or collect preliminary data and fit them to a statistical distribution.

Although the greater flexibility of the negative binomial makes it an ideal candidate, I recommend that all distributional assumptions be explicitly tested.

The greater mobility of fish also allowed them to evade capture and likely influenced the observed negative relationship between density and dispersion. Sampling efficiency for the sessile organisms (i.e., aquatic benthic invertebrates and plants) was presumably very close to $100 \%$, whereas relative sampling efficiency averaged less than $35 \%$ across salmonid species (Table 2). The inability to count or capture all of the individuals in a sampling unit would have increased variance regardless of fish density. The greater accuracy of the beta-binomial, which accounts for extra variation (overdispersion) relative to the binomial, also suggests that variability in sampling efficiency influenced data dispersion (i.e., negative binomial $\underline{\mathrm{k}}$ ). Overdispersion was probably the result of a combination of factors including failure to incorporate significant influences on efficiency into the sampling efficiency models (e.g., the effects of fish size) and the non-independence of species' responses (Bayley 1993). Among these, the nonindependence of fish response would have a greater effect on the relationship between density and dispersion. For example, fishes fleeing from a sampling crew are likely to affect the behavior of other fish (e.g., flight, concealment) in the sampling unit. In effect, several fish act as single individual (their responses are dependent) and depending upon their joint response, none are captured (0\% efficiency) or all are captured (100\% 
efficiency). When fish abundance is high, there is likely a greater number of fish acting as an individual. This would increase variance (i.e., decrease $k$ ) for higher fish densities and result in a negative relationship between density and negative binomial dispersion. Therefore, the positive relationship between actual density and dispersion was probably greater than that observed for the raw catch data.

Presumably, detection probabilities will be used to develop sample size requirements for surveying or monitoring rare or endangered species (e.g., Bonar et al. 1997). In these instances, regulatory agencies would likely be required to choose target or threshold densities for estimating detection probabilities (Green and Young 1993). The choice of a biologically meaningful threshold is wrought with uncertainty, especially for a rare species. The use of a gamma prior distribution is one means to incorporate this uncertainty into sampling guidelines. Indeed, the Poisson-gamma detection probability and sample size estimates were relatively accurate (Table 3), which suggests that the gamma could provide a realistic approximation of the variability of fish density (e.g., minimum viable population size) or uncertainty about a biologically meaningful threshold. For example, existing data on distribution and abundance of target species could be combined and fitted to a gamma distribution. This would provide the most biologically realistic measure of the variation. Where existing data are lacking, a prior could be developed from a distribution of expert opinions on the minimum density to maintain a viable population (i.e., a subjective prior; Gelman et al. 1995). The data generated from the subsequent monitoring or surveys then can be used to update the prior and reexamine the distributional assumptions. 
The widespread use and acceptance of the Poisson distribution to estimate detection probabilities and sample size requirements was probably due, in part, to Green and Young's (1993: 356) contention that the adequacy of the Poisson estimator "do[es] not depend on species, habitat, sampling method, or sample unit size." Yet as I indicated above, these are the same factors affecting the variability of sampling efficiency and fish distribution. To be fair, Green and Young suggested that preliminary sampling would be useful for examining the adequacy of the Poisson assumption. My results, however, suggest that preliminary data are not just useful, but necessary for developing rigorous, defensible survey and monitoring protocols. Failure to examine the adequacy of Poisson, negative binomial, or other models before adopting a protocol can result in the collection of useless data. Worse, "a poorly performed survey means the inertia of its imprecise results must be overcome before a legitimate survey can be conducted" (Thompson et al. 1998: xii). In these instances, the additional costs associated with overcoming the inertia and renovating the survey design would very likely exceed any costs that would have been incurred during preliminary sampling and analyses. Therefore, I strongly recommend that all monitoring or survey protocols include a pilot or implementation phase that allows for periodic evaluations of the data so that assumptions can be assessed and sampling strategies revised, if necessary. 


\section{Acknowledgments}

Several people were instrumental in compiling and creating the fish collection databases. I am particularly indebted to Kerry Overton and Sherry Wollrab for the development and management of the FS survey database; Debby Myers and Gwynne Chandler for quality control and management of the monitoring database; and Julie Richardson, Sharon Parkes, Dona Horan, Terry Elms-Cochran, and Judy Hall- Griswold for development and validation of the monitoring data. The manuscript was improved with suggestions from Bill Thompson, Peter Bayley, Gary Grossman, Bruce Rieman, and Jason Dunham. This work was funded by the Bonneville Power Administration under project $92-32$. 


\section{Literature Cited}

Anderson, C. 1995. Measuring and correcting for size selection in electrofishing markrecapture experiments. Trans. Am. Fish. Soc. 124:663-676.

Angermeier, P. L. and J. R. Karr. 1984. Relationships between woody debris and fish habitat in a small warmwater stream. Trans. Am. Fish. Soc. 113, 716-726.

Baltz, D. M., P. B. Moyle, and N. J. Knight. 1982. Competitive interactions between

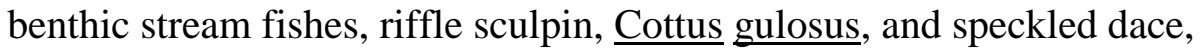

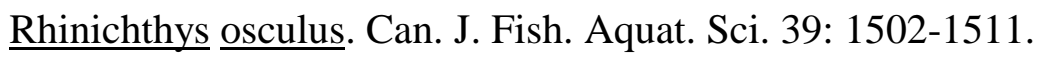

Baltz, D. M., B. Vondracek, L. R. Brown, and P. B. Moyle. 1991. Seasonal changes in microhabitat selection by rainbow trout in a small stream. Trans. Am. Fish. Soc. 120: 166-176.

Bayley, P. B. 1993. Quasi-likelihood estimation of marked fish recapture. Can. J. Fish. Aquat. Sci. 50: 2077-2085.

Bisson, P. A., K. Sullivan, and J. L. Nielson. 1988. Channel hydraulics, habitat use, and body form of juvenile coho salmon, steelhead, and cutthroat trout. Trans. Am. Fish. Soc. 117: 262-273.

Bliss, C. I., and R. A. Fisher. 1953. Fitting the negative binomial distribution to biological data a note on the efficient fitting of the negative binomial. Biometrics, 9: 176-200.

Berger, J. O. 1985. Statistical decision theory and Bayesian analysis. Springer-Verlag, New York. 
Bonar, S.A., M. Divens, and B. Bolding. 1997. Methods for sampling the distribution and abundance of bull trout/ dolly varden. Wash. Dept. Fish Wildl. Res. Rep. RAD9705

Buttiker, B. 1992. Electrofishing results corrected by selectivity functions in stock size estimates of brown trout ( $\underline{\text { Salmo }} \underline{\text { trutta }}$ L.) in brooks. J. Fish Bio. 41:673-684.

Dolloff, A., J., Kershner, and R. Thurow. 1996. Underwater observation. In Fisheries Techniques, second edition. Edited by B.R. Murphy and D.W. Willis. American Fisheries Society, Bethesda, Maryland. pp. 533-553.

Dunham, J. B., and B. E. Rieman. 1999. Metapopulation structure of bull trout: influences of habitat size, isolation, and human disturbance. Ecol. Apps. 9: 642655.

Edmundsen, E., F. E. Everest, and D.W. Chapman. 1968. Permanence of station in juvenile chinook salmon and steelhead trout. J. Fish. Res. Board Can. 25: 14531464.

Elliott, J.M. 1969. Some methods for the statistical analysis of samples of benthic invertebrates. Freshwater Biol. Assoc. Sci. Pub. No. 25.

Fraser, D. F., D. A. DiMattia, and J. D. Duncan. 1987. Living among predators: the response of a stream minnow to the hazards of predation. In Community and Evolutionary Ecology of North American Stream Fishes. Edited by W.S. Matthews and D. C. Heins. Oklahoma University Press, Norman Okla. pp 97-123.

Gelman, A.B., J.S. Carlin, H.S. Stern, and D.B. Rubin. 1995. Bayesian data analysis. Chapman and Hall, Boca Raton. 
Gorman, O. T., and J. R. Karr. 1978. Habitat structure and stream fish communities. Ecology, 59: 507-515.

Gowan, C., M. K. Young, K. D. Fausch, and S. C. Riley. 1994. Restricted movement in resident stream salmonids: a paradigm lost? Can. J. Fish. Aquat. Sci. 51: 26262637.

Green, R. H., and R. C. Young. 1993. Sampling to detect rare species. Ecol. Apps. 3: 351-356.

Greig-Smith. P. 1964. Quantitative plant ecology. Butterworths, London.

Hariston, N. G. 1959. Species abundance and community organization. Ecology, 40: 404416.

Hillborn, R., and M. Mangel. 1997. The ecological detective- confronting models with data. Princeton University Press, Princeton.

Hillman, T. W., and W. S. Platts. 1993. Survey plan to detect the presence of bull trout. Technical Report. Don Chapman Consultants Incorporated, Boise, Idaho.

Matthews, W. J., and H. W. Robinson. 1988. The distribution of fish in Arkansas. Copeia, 3: 58-374.

Osborne, L. L., and M. J. Wiley. 1992. Influence of tributary spatial position on the structure of warmwater fish communities. Can. J. Fish. Aquat. Sci. 49: 671-681.

Overton, C. K, J. D. McIntyre, R. Armstrong, S. L. Whitwell, and K. A. Duncan. 1995. Users guide to fish habitat: descriptions that represent natural conditions in the Salmon River basin, Idaho. U.S. For. Serv. Gen. Tech. Rep. INT-GTR-345. 
Overton, J. M., T. C. Young, and W. S. Overton. 1993. Using found data to augment a probability sample: procedure and case study. Environ. Monitor. Assess. 26: 6583.

Peterson, J. T., and S. P. Wollrab. 1999. An analysis of potential stream fish and fish habitat monitoring procedures for the Inland Northwest. Report submitted to Bonneville Power Administration, Portland, Oregon. DOE/BP-25866-1.

Petty, J. T., and G. D. Grossman. 1996. Patch selection by mottled sculpin (Pisces: Cottidae) in a southern Appalachian stream. Freshwater Biol. 35: 261-276.

Pielou, E.C. 1969. An introduction to mathematical ecology. Wiley. New York. Poole, R.W. 1974. An introduction to quantitative ecology. McGraw-Hill. New York.

Prentice, R.L. 1986. Binary regression using an extended beta-binomial distribution with discussion of correlation induced by covariate measurement errors. J. Am. Stat. Assoc. 81:321- 327.

Reynolds, J. B. 1996. Electrofishing. In Fisheries Techniques, second edition. Edited by B.R. Murphy and D.W. Willis. American Fisheries Society, Bethesda, Maryland. pp. 221-254.

Rieman, B., J. Dunham, and J. Peterson. 1999. Development of a database to support a multi-scale analysis of the distribution of westslope cutthroat trout. Final Report to U.S. Geological Survey, Reston, Virginia. 1445-HQ-PG-01026.

Rieman, B. E., and J. D. McIntyre. 1995. Occurrence of bull trout in naturally fragmented habitat patches of varied size. Trans. Am. Fish. Soc. 124: 285-296.

Thompson, W. L., G. C. White, and C. Gowan. 1998. Monitoring vertebrate populations. Academic Press, New York. 
Thurow, R. F., D. C. Lee, and B. E. Rieman. 1997. Distribution and status of seven native salmonids in the interior Columbia River Basin and portions of the Klamath River and Great basins. N. Am. J. Fish. Manage. 17:1094-1110.

Scott, W. B., and E. J. Crossman. 1973. Freshwater fishes of Canada. Bull. Fish. Res. Board Can. No. 184.

Shaw, E. 1978. Schooling fishes. Am. Scient. 66:166-175.

Sokal, R. R., and J. Rohlf. 1995. Biometry: the principles and practice of statistics in biological research, third edition. W.H. Freeman, San Francisco.

Southwood, T.R.E. 1966. Ecological methods: with particular reference to the study of insect populations. Methuen, London.

Strayer, D.L. 1999. Statistical power of presence-absence data to detect population declines. Cons. Biol. 13: 1034-1038.

Wang, P., M. L. Putterman, I. Cockburn, and N. Le. 1996. Mixed Poisson regression models with covariate dependent rates. Biometrics, 52:381- 400.

Watson, G., and T.W. Hillman. 1997. Factors affecting the distribution and abundance of bull trout: an investigation at hierarchical scales. N. Am. J. Fish. Manage. 17:237246.

White, G.C., and R.E. Bennetts. 1996. Analysis of frequency count data using the negative binomial distribution. Ecology, 77: 2549-2557. 
Table 1. Means, standard errors (in parenthesis) and range of mean density (number per sample unit) and the negative binomial dispersion parameter $(\underline{\mathrm{k}})$, by species, and coefficients, standard errors (SE), and 95\% confidence limits (CL) from linear regression of species-specific mean abundance and negative binomial dispersion parameter. The dispersion parameter was natural log transformed prior to fitting the linear model and steelhead/ redband trout was the baseline category for the regression.

\begin{tabular}{|c|c|c|c|c|}
\hline$\underline{\text { Species }}$ & $\underline{N}^{1}$ & \multicolumn{2}{|c|}{ Density } & $\underline{\mathrm{k}}$ \\
\hline Bull trout & 22 & \multicolumn{2}{|c|}{$0.409(0.335)$} & $16.085(39.137)$ \\
\hline (Salvelinus confluentus) & & \multicolumn{2}{|c|}{$0.02-1.33$} & $0.03-177.95$ \\
\hline Brook trout & 32 & \multicolumn{2}{|c|}{$2.445(3.116)$} & $1.912(4.403)$ \\
\hline (Salvelinus fontinalis) & & \multicolumn{2}{|c|}{$0.05-12.40$} & $0.04-18.46$ \\
\hline Westslope cutthroat trout & 26 & \multicolumn{2}{|c|}{$1.452(0.279)$} & $2.283(0.439)$ \\
\hline (Oncorhynchus clarki lewisi) & & \multicolumn{2}{|c|}{$0.04-8.00$} & $0.07-34.87$ \\
\hline Chinook salmon parr & 27 & \multicolumn{2}{|c|}{$4.032(5.131)$} & $2.627(8.89)$ \\
\hline (Oncorhynchus tshawytscha) & & \multicolumn{2}{|c|}{$0.09-19.61$} & $0.04-40.05$ \\
\hline Steelhead /redband trout & 41 & \multicolumn{2}{|c|}{$2.804(3.598)$} & $10.741(47.548)$ \\
\hline (Oncorhynchus mykiss spp.) & & \multicolumn{2}{|c|}{$0.04-14.00$} & $0.08-304.97$ \\
\hline$\underline{\text { Parameter }}$ & $\underline{\text { Coefficient }}$ & $\underline{\mathrm{SE}}$ & ${\underline{\text { Upper }} \mathrm{CL}^{2}}^{2}$ & $\underline{\text { Lower CL }}$ \\
\hline Intercept & -0.095 & 0.317 & 0.527 & -0.717 \\
\hline Density & 0.149 & 0.066 & 0.277 & 0.020 \\
\hline Bull trout & 0.501 & 0.556 & 1.590 & -0.588 \\
\hline Brook trout & -0.340 & 0.533 & 0.674 & -1.413 \\
\hline Westslope cutthroat trout & -0.616 & 0.503 & 0.370 & -1.601 \\
\hline Chinook salmon & -1.077 & 0.549 & -0.001 & -2.152 \\
\hline Density*Bull trout & 0.273 & 0.871 & 1.981 & -1.434 \\
\hline Density*Brook trout & -0.127 & 0.123 & 0.114 & -0.367 \\
\hline Density*Westslope cutthroat trout & 0.138 & 0.182 & 0.495 & -0.218 \\
\hline Density*Chinook salmon & -0.130 & 0.100 & 0.066 & -0.326 \\
\hline
\end{tabular}

${ }^{1}$ Number of reaches for which a mean and dispersion parameter were estimated.

${ }^{2}$ Confidence limits can be used to infer statistical significance at $\underline{\mathrm{P}}<0.05$. 
Table 2. Average habitat characteristics, fish abundance, and relative sampling efficiency (\%) for monitoring sites used for the comparison of 4 approaches to estimating detection probabilities, for bull and westslope cutthroat trout.

\begin{tabular}{|c|c|c|c|c|c|c|c|c|c|}
\hline & & & & Mean & Mean & & & $\begin{array}{l}\text { Relative } \\
\text { sampling }\end{array}$ & $\begin{array}{c}\text { Beta-binomial } \\
\text { dispersion }\end{array}$ \\
\hline$\underline{\mathrm{N}}$ & & $\underline{\text { Elevation }(\mathrm{m})}$ & Length (m) & $\underline{\text { width }(\mathrm{m})}$ & depth $(m)$ & Gradient $(\%)$ & Abundance & $\underline{\text { efficiency }}^{1}$ & parameter \\
\hline \multicolumn{10}{|l|}{ Bull trout } \\
\hline 74 & mean & 1485 & 101.6 & 14.4 & 0.34 & 0.83 & 5.2 & 25.0 & 0.5055 \\
\hline & SE & 51.2 & 4.53 & 1.02 & 0.017 & 0.049 & 0.85 & 1.69 & \\
\hline & range & $343-2110$ & $39.5-280.6$ & $3.7-48.6$ & $0.08-0.91$ & $0.05-2.10$ & $1-34$ & $7.06-87.0$ & \\
\hline \multicolumn{10}{|l|}{ Brook trout } \\
\hline 73 & mean & 1596 & 101.5 & 13.1 & 0.31 & 0.76 & 36.3 & 31.3 & 0.3633 \\
\hline & SE & 49.4 & 4.89 & 0.892 & 0.016 & 0.068 & 8.82 & 1.85 & \\
\hline & range & $343-2110$ & $39.5-280.6$ & $3.7-47.8$ & $0.08-0.91$ & $0.02-4.10$ & $1-386$ & $7.6-92.5$ & \\
\hline \multicolumn{10}{|c|}{ Westslope cutthroat trout } \\
\hline 92 & mean & 1382 & 95.5 & 14.7 & 0.34 & 0.82 & 16.4 & 34.8 & 0.3804 \\
\hline & SE & 47.2 & 4.03 & 0.854 & 0.014 & 0.060 & 2.89 & 1.62 & \\
\hline & range & $343-2024$ & $39.2-280.6$ & $3.8-48.6$ & $0.08-0.91$ & $0.05-4.10$ & $1-164$ & $8.2-92.3$ & \\
\hline
\end{tabular}

\footnotetext{
${ }^{1}$ Sampling efficiency estimates are relative to removal estimates and are probably biased high.
} 
Table 3. Mean density, variances $\left(\underline{s^{2}}\right)$, gamma shape $(\underline{a})$ and scale $(\underline{b})$ parameters, negative binomial dispersion parameter $(\underline{\mathrm{k}})$, probability of detection estimates, and sample size requirements for $95 \%$ power (in parenthesis), by species, for salmonids collected during FS survey of small streams in the Interior Columbia River Basin. Probabilities of detection were estimated using the Poisson, Poisson-gamma, and negative binomial estimators. Baseline is the proportion of the 1271 sample units that each species was detected and is shown for comparison.

\begin{tabular}{|c|c|c|c|c|c|c|c|c|c|}
\hline \multirow[b]{2}{*}{ Species } & \multirow[b]{2}{*}{ Density } & \multirow[b]{2}{*}{$\underline{s}^{2}$} & \multirow[b]{2}{*}{$\underline{\mathrm{a}}$} & \multirow[b]{2}{*}{$\underline{\mathrm{b}}$} & \multirow[b]{2}{*}{$\underline{\mathrm{k}}$} & \multicolumn{4}{|c|}{ Probability of detection } \\
\hline & & & & & & $\underline{\text { Baseline }}$ & $\underline{\text { Poisson }}$ & $\begin{array}{l}\text { Poisson- } \\
\text { gamma }\end{array}$ & $\begin{array}{l}\text { Negative } \\
\text { binomial }\end{array}$ \\
\hline \multicolumn{10}{|c|}{ Bull trout } \\
\hline & 0.257 & 0.599 & 1.272 & 0.195 & 0.169 & $0.150(18)$ & $0.227(12)$ & $0.148(19)$ & $0.145(19)$ \\
\hline \multicolumn{10}{|c|}{ Brook trout ${ }^{1}$} \\
\hline & 0.906 & 18.481 & 10.680 & 0.085 & 0.048 & $0.127(22)$ & $0.596(3)$ & $0.188(14)$ & $0.134(21)$ \\
\hline \multicolumn{10}{|c|}{ Westslope cutthroat trout } \\
\hline & 0.600 & 4.133 & 4.958 & 0.121 & 0.108 & $0.154(18)$ & $0.451(5)$ & $0.194(14)$ & $0.184(15)$ \\
\hline \multicolumn{10}{|c|}{ Chinook salmon $^{1}$} \\
\hline & 1.553 & 73.340 & 18.551 & 0.084 & 0.034 & $0.103(28)$ & $0.788(2)$ & $0.220(12)$ & $0.121(23)$ \\
\hline \multicolumn{10}{|c|}{ Steelhead/ redband trout } \\
\hline & 1.639 & 17.281 & 11.979 & 0.137 & 0.172 & $0.344(7)$ & $0.806(2)$ & $0.296(9)$ & $0.333(7)$ \\
\hline
\end{tabular}




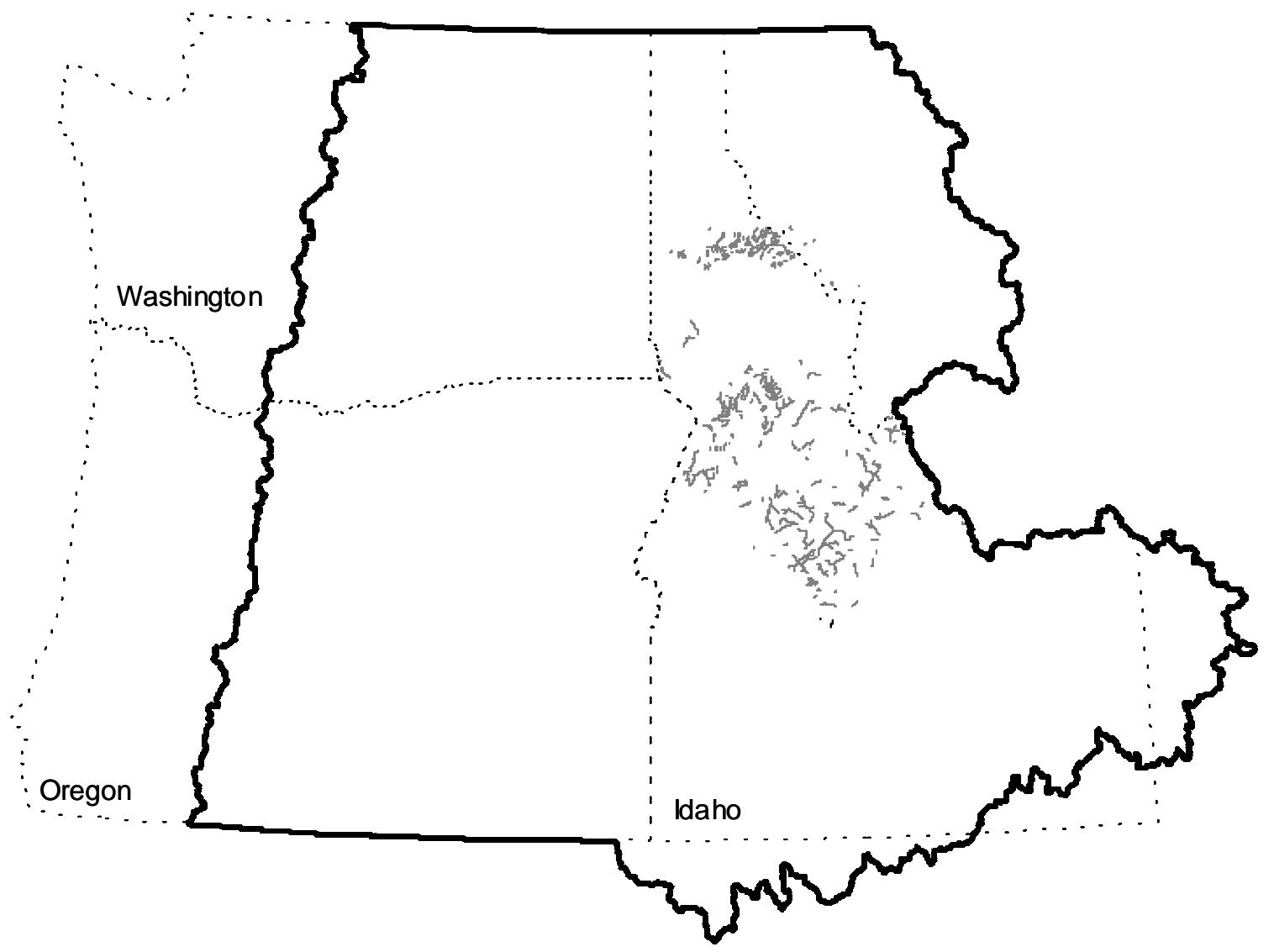

Figure 1. Locations of surveyed and monitored stream channels (gray lines) in the interior Columbia River Basin (bold outline). States are labeled and boundaries (broken line) are shown for reference. 

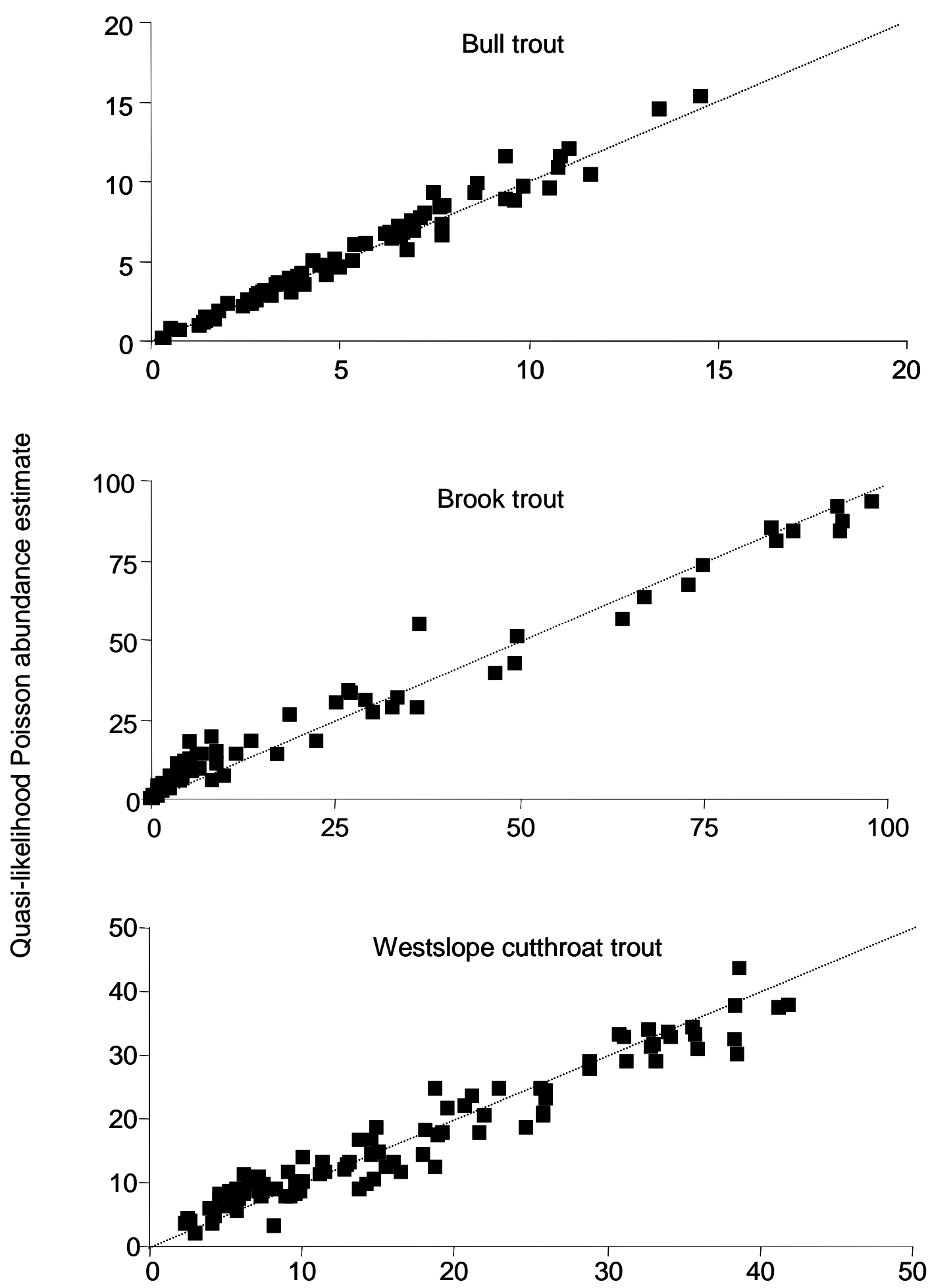

Negative binomial abundance estimate

Figure 2. Abundance estimates from the quasi-likelihood Poisson and negative binomial regressions for (a) bull trout, (b) brook trout, and (c) westslope cutthroat trout calculated during the leave-one-out cross-validation procedure. Broken line represents perfect fit. 


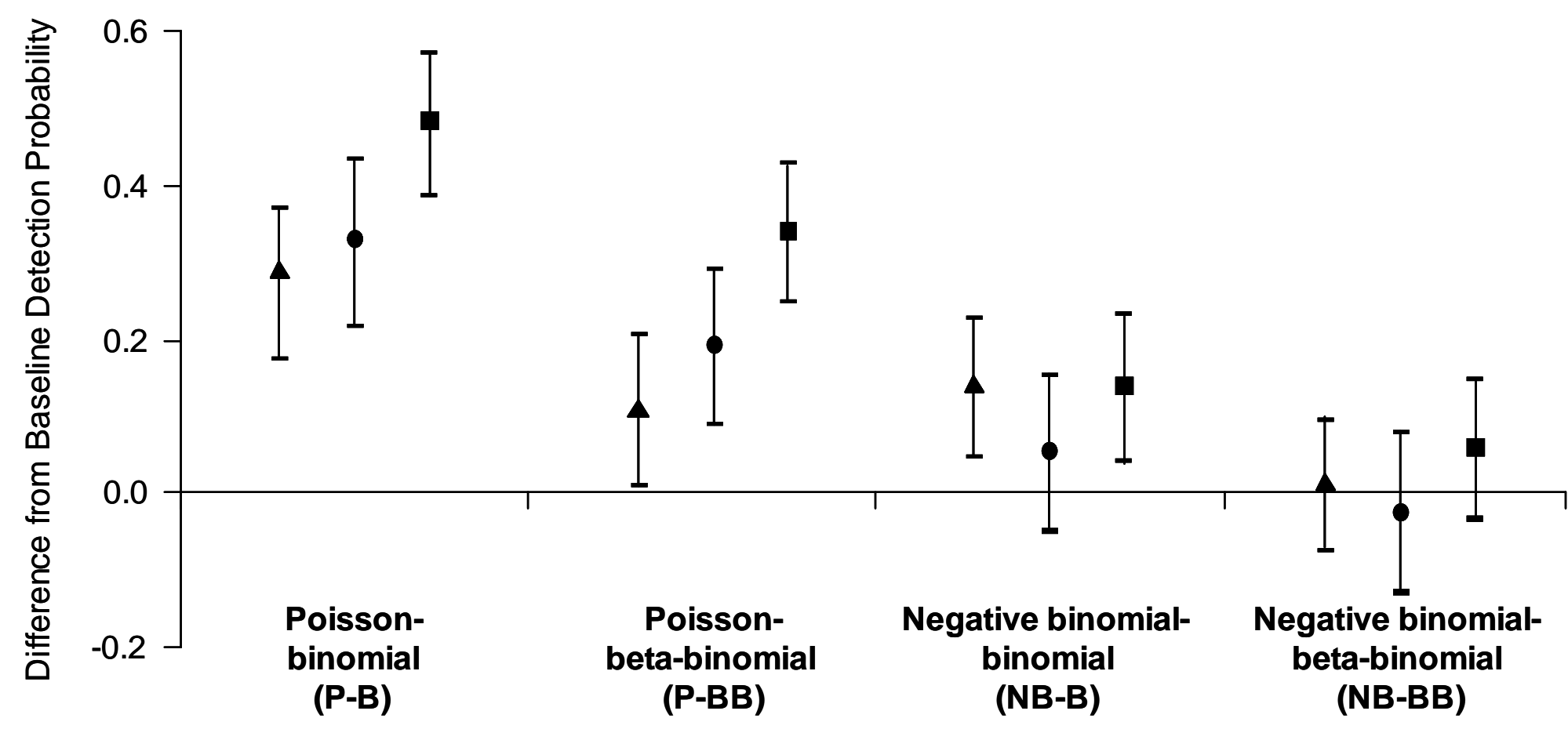

Figure 3. The mean differences between baseline detection probabilities and four detection probability estimators for bull trout (triangle), brook trout (circle), and westslope cutthroat trout (square). Brackets are $95 \%$ confidence intervals. 